\title{
Smarter Medicine - Choosing Wisely Switzerland
}

\author{
Der schwierige Weg vom Slogan «Mehr tun bedeutet \\ nicht immer, das Beste zu tun» zur Änderung
}

\author{
Luca Gabutti und Angela Greco² \\ 'Ente Ospedaliero Cantonale, Bellinzona; Mitglied des Gremiums für Smarter Medicine - Choosing Wisely Switzerland. \\ ${ }^{2}$ Qualität und Patientensicherheit, Ente Ospedaliero Cantonale, Locarno
}

Smarter Medicine - Choosing Wisely Switzerland ist eine im Jahr 2014 gegründete Vereinigung, deren Ziel in der Förderung der Kampagne Choosing Wisely besteht [1]. Die Schweiz nimmt auch an den Aktivitäten von Choosing Wisely International teil und im Jahr 2018 war sie in Zürich Gastgeber für die Delegierten der etwa 20 Länder von fünf Kontinenten, die ihr offiziell angehören [2,3]. Bis heute haben zwölf Fachgesellschaften ihre Top-5-Liste der medizinischen Verfahren veröffentlicht, die gemäss dem Slogan «Mehr tun bedeutet nicht immer, das Beste zu tun» zu vermeiden sind, und einige Ergebnisse der auf den Empfehlungen beruhenden Massnahmen wurden veröffentlicht $[1,4-7]$.

Das Ziel dieser Sammlung von Artikeln besteht gerade darin, die Hauptakteure durch ihr Fachunternehmen aufzufordern, über ihre Erfahrungen zu berichten, wobei sie insbesondere auf die Veränderungen eingehen, die durch die Kampagne herbeigeführt wurden.

Sicherlich fragen Sie sich, warum wir über die Übermedikalisierung sprechen. Warum ausgerechnet in einer Zeit, in welcher uns der Lockdown und die Angst der Patienten vor einer Ansteckung mit COVID-19 die negativen Auswirkungen fehlender unerlässlicher Behandlungen vor Augen führen. In einer Zeit, in der die Schweizerische Gesellschaft für Allgemeine Innere Medizin und die Schweizerische Gesellschaft für Kardiologie sogar die Patienten an die Wichtigkeit erinnern, bei Auftreten bestimmter Symptome, sofort ärztliche Hilfe zu suchen [8, 9].

Wir sind dennoch der Ansicht, dass der Zusammenbruch der Gesundheitsdienstleistungen in der akuten Phase der Pandemie uns veranlassen sollte, auch über die Tragweite der Übermedikalisierung nachzudenken. Nie zuvor konnten wir uns darüber bewusstwerden, wie überflüssig sie ist. Die Feststellung, was unentbehrlich ist, ist nicht immer einfach, da es nicht nur zu verstehen gilt, welche Behandlungen nicht notwendig sind, sondern auch festgestellt werden muss, welche Behandlungen im Hinblick auf die Gesundheit einen geringen Wert für die Patienten haben. Wenn wir von Wert sprechen, denken wir an das Kosten-Nutzen-Verhältnis, wobei sowohl finanzielle Aspekte (mit Krankenkassenprämien, die nicht mehr allen zugänglich sind) als auch Nebenwirkungen (das Antibiotikum, das im Falle einer Lungenentzündung lebensrettend ist, hat einen wesentlichen höheren Wert als die Knieprothese, bei der es aufgrund einer Infektion eines Patienten, der an einer leichten Arthrose litt, zu Komplikationen kommt) berücksichtigt werden.

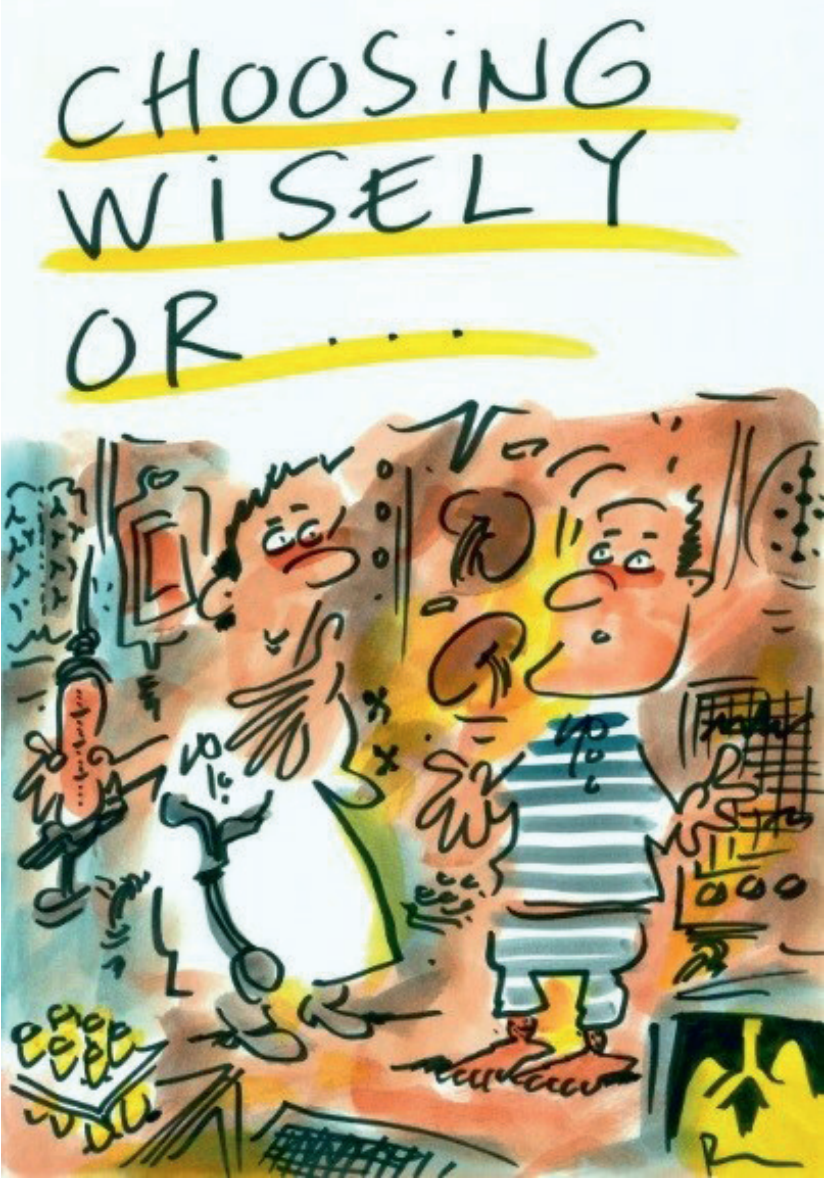

Illustration von Pecub (Piepaolo Pugnale) zum Thema Choosing Wisely. 
Es ist jedoch unnötig, sich lange mit dem Warum aufzuhalten; niemand von uns bezweifelt ernsthaft die Tatsache, dass man sich weniger auf das Tun und stärker auf die Ergebnisse konzentrieren sollte. Die offene Frage ist, wie dies zu erreichen ist.

Was brauchen wir, um die Veränderung herbeizuführen? Müssen wir vielleicht nochmal eine schöne Review über Choosing Wisely lesen oder uns die Empfehlungen, was nicht getan werden sollte, noch einmal ansehen?

Das glauben wir nicht; wir sind eher der Ansicht, dass es wichtig ist, sich mit dem Wie zu beschäftigen und etwas Zeit zu investieren, um beispielsweise einen Artikel über die Methode des Wandels zu lesen, der vor einigen Monaten von Compton-Phillips veröffentlicht wurde [10].

Unter Bezugnahme auf die fünf Schlüsselwörter Vision, Trust, Data, Capacity und Alignement erinnert der Verfasser daran, dass Folgendes erforderlich ist, um ein Projekt im Gesundheitswesen zu Ende zu führen: Eine Vision (das heisst Ermittlung des Problems, das behoben werden soll), der Glaube daran (Erzielung eines Konsensus, Einbeziehung insbesondere der Schlüsselpersonen des Netzes), Verfügbarkeit von relevanten Daten (mit Überwachung der Veränderung), die entsprechende Fähigkeit (Bereitstellung der notwendigen Instrumente für das klinische Personal) und Anpassung der Anreize an die erwartete Verhaltensweise (Belohnung des medizinischen Fachpersonals für die erzielten Ergebnisse und nicht für die erbrachten Leistungen).

Ohne Zweifel hat sich die medizinische Forschung der letzten Jahrzehnte sehr positiv auf die Gesundheit ausgewirkt; eine klare Tendenz zur Überversorgung und, damit verbunden, Ressourcenverschwendung sind hingegen unerwünscht. Der «smarte» Arzt sollte sich deshalb vor jeder Behandlung folgende drei Fragen stellen: Kann die Behandlung schädlich sein? Gibt es Evidenz, dass die Behandlung wirkt? Ist die Behandlung nötig? Die Behandlung orientiert sich somit an den individuellen Bedürfnissen und wird dadurch nachhaltiger und sozial gerechter.

Wir danken daher allen Kollegen, die mit Leidenschaft und Engagement an dieser Ausgabe der Therapeutischen Umschau, die Smarter Medicine - Choosing Wisely gewidmet ist, mitgewirkt haben.

\section{Literatur}

1. Smarter medicine [Internet]. Bern: Schweizerische Gesellschaft für Allgemeine Innere Medizin (SGAIM); c2021 [abgerufen am 20. Juni 2020]. Verfügbar unter: https://www.smarter medicine.ch/de/home.html

2. Levinson W, Kallewaard M, Bhatia RS, Wolfson D, Shortt S, Kerr EA. 'Choosing Wisely': a growing international campaign, BMJ Qual Saf. 2015:24:167- 74 .

3. ABIM Foundation, Hrsg. Choosing Wisely Around the World [Internet]. Philadelphia: ABIM Foundation; 14. Mai 2015 [abgerufen am 20. Juni 2020]. Verfügbar unter: https://www. choosingwisely.org/resources/updates-from-the-field/choos ing-wisely-around-the-world/

4. Del Giorno R, Ottini A, Greco A, Stefanelli K, Kola F, Clivio L, et al. Peer-pressure and overuse: The effect of a multimodal approach on variation in benzodiazepine prescriptions in a network of public hospitals. Int J Clin Pract. 2020;74(3):e13448.

5. Del Giorno R, Ceschi A, Pironi M, Zasa A, Greco A, Gabutti L. Multifaceted intervention to curb in-hospital over-prescription of proton pump inhibitors: A longitudinal multicenter quasi-experimental before-and-after study. Eur J Intern Med. 2018;50:52-59.

6. Erard Y, Del Giorno R, Zasa A, De Gottardi S, Della Bruna R, Keller $F$, et al. A multi-level strategy for a long lasting reduction in unnecessary laboratory testing: A multicenter before and after study in a teaching hospital network. Int $J$ Clin Pract. 2018;73(3):e13286.

7. Del Giorno R, Greco A, Zasa A, Clivio L, Pironi M, Ceschi A, et al. Postgrad Combining prescription monitoring, benchmarking, and educational interventions to reduce benzodiazepine prescriptions among internal medicine inpatients; a multicenter before and after study in a network of Swiss Public Hospitals. Postgrad Med. 2018;130:627 - 636.

8. SGAIM. Trotz SARS-CoV-2: Die Grundversorgung ist garantiert [Medienmitteilung] (8. April 2020). Verfügbar unter: https:// www.sgaim.ch/nc/fr/meta/news/detail/news/trotz-sars-cov2-die-grundversorgung-ist-garantiert.html

9. Fondation Suisse de Cardiologie. En cas de soupcon d'infarctus du myocarde ou d'AVC, il faut appeler les urgences au 144 , y compris en cette période de crise due au coronavirus [Medienmitteilung] (3. April 2020). Verfügbar unter: https://www. swissheart.ch/nc/fr/actualite-evenements/actualites/newsdetail-fr/news/en-cas-de-soupcon-dinfarctus-du-myocardeou-davc-il-faut-appeler-les-urgences-au-144-y-compris-e. html

10. Compton-Phillips A, Spreading at Scale: A Practical Leadership Model for Change. NEJM Catalyst. 2019;01(01).

\section{Prof. Luca Gabutti}

Chefarzt der Inneren Medizin

Ospedale San Giovanni

Via Ospedale 12

6500 Bellinzona

luca.gabutti@eoc.ch 